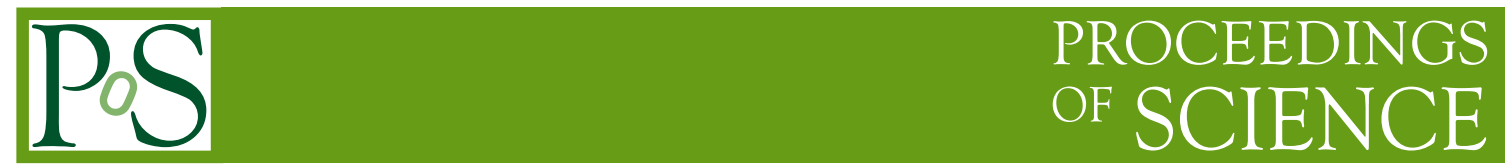

Highlights and perspectives from the CMS experiment

\author{
Gautier Hamel de Monchenault ${ }^{a}{ }^{\dagger}$ \\ ${ }^{a} I R F U, C E A$, Université Paris-Saclay, \\ Gif-sur-Yvette, France \\ $\dagger$ on behalf of the CMS Collaboration \\ E-mail: Gautier.Hamel.de.Monchenault@cern.ch
}

Highlights of the CMS experience are presented. A status of the detector installation in preparation for the LHC Run-3, which will start in early 2022, is given. Some of the key CMS physics results of the past year are recalled. The most recent results, included those presented at this conference, are also mentioned. A brief description of the ongoing CMS upgrade activities is provided.

The Ninth Annual Conference on Large Hadron Collider Physics - LHCP2021

7-12 June 2021

Online 


\section{Introduction: status of the experiment}

Since 2018, the CMS detector [1] is in its maintenance and upgrade phase, called the Long Shutdown 2 (LS2). LS2 activities continue as planned, with only minor delays mainly due to periods of confinement and travel restrictions. Some LS2 upgrade activities to highlight are:

- the replacement of the front-end electronics of the CSC muon chambers in the end-cap regions, needed in sustain HL-LHC trigger rates. This major LS2 work was completed in 2020, with few months delay due to the pandemic;

- the installation of a new layer of high-granularity muon chambers based on Gaz Electron Multipliers (GEM), complementing the first station of muon chambers in the end-cap regions. This GEM layer, which covers the pseudo-rapidity range from 1.6 to 2.1, is the first Phase-2 upgrade detector installed in CMS. Together with another layer, to be integrated during LS3 to complement the second station, the GEM muon chambers will allow a reduction of the L1 trigger rate on muons in the forward regions by a factor of at least 10, as well as an improved trigger efficiency on forward displaced muons;

- the completion of the Phase-1 upgrades of the hadronic calorimeter (HCAL). These consisted in the replacement of the previous readout based on hybrid photo-diodes by a more efficient readout based on silicon photomultipliers, with a much improved longitudinal segmentation both in the barrel and end-cap regions;

- the installation of a much lighter beam pipe, completely redesigned for the high luminosity phase of the LHC. The new CMS beam pipe was fully aligned and leak tested, and the bake-out was completed the week before the start of the conference. This crucial milestone for CMS constitutes the biggest upgrade of the experiment's vacuum system since 2008;

- the refurbishment of the pixel detector, including a new barrel layer closer to the interaction point, with a more radiation tolerant electronics, and the replacement of all the DCDC converters. The re-installation of the pixel detector in CMS was completed successfully a couple of weeks after the conference;

- the successful implementation of a heterogeneous computing architecture in the High Level Trigger (HLT), with a mixture of GPUs and CPUs, already yielding significant speed improvement.

The CMS magnet is expected to be fully commissioned at 3.8 $\mathrm{T}$ for the two-week Pilot Beam Test run scheduled on the second part of October 2021. Then, the experiment will be re-opened for relatively minor interventions, including the installation of muon chamber demonstrators for HL-LHC. CMS is fully on track for the restart of the LHC for Run-3 in February 2022, after several weeks of commissioning with cosmic rays at nominal magnetic field (CRAFT).

The goal of Run-3 is to collect proton-proton (pp) collision data at $\sqrt{s}=13.6 \mathrm{TeV}$ with similar pileup conditions than in 2018, as well as heavy ion collision data. The Run-3 pp dataset is expected to be equivalent to that of Run-2 in integrated luminosity, but with enhanced trigger capabilities. Furthermore, the analysis methods have considerably improved in recent years, with the generalised 
usage of advanced machine learning techniques, capable of boosting the sensitivity of physics analyses. From CMS perspective, the physics case for Run-3 is considerable.

\section{Physics results}

The physics output of CMS is unprecedented in high-energy physics. At the date of the conference (June 2021), a total of 1043 papers based on collision data had been published (or submitted) by CMS, beyond the symbolic number of one thousand. In addition to standard high energy physics journal publications, several CMS papers have now been published in dedicated machine learning journals, illustrating the development of such tools inside the collaboration. Most recent publications are based on the entire Run 2 data set (data taken in years 2016-2018), corresponding to a total integrated luminosity of $137 \mathrm{fb}^{-1}[2,3]$.

As many as seventeen preliminary physics results were presented for the first time at the conference [4-20]; they are described in detail elsewhere in these proceedings. Here, we highlight some results published or released since the last edition of the conference.

\subsection{Highlights on standard model studies}

In a paper published in 2020 [21], CMS has conducted a thorough study of W-boson production and decay in the muonic channel with 2016 data $\left(13 \mathrm{TeV}, 36 \mathrm{fb}^{-1}\right)$. The measurement of doubledifferential cross sections in 19 bins of muon transverse momentum $\left(p_{\mathrm{T}}^{\mu}\right)$ between 26 and $45 \mathrm{GeV}$ and 48 bins of pseudo-rapidity $\left(\eta^{\mu}\right)$ between -2.4 and 2.4, are used to extract differential crosssections and charged asymmetries separately for two the W-boson helicity states, $\mathrm{W}_{\mathrm{L}}$ and $\mathrm{W}_{\mathrm{R}}$, which are compared to predictions at the next-to-leading order (NLO) in QCD. These results help constrain parton density functions (PDFs) and constitute an important milestone towards the eventual measurement of the W-boson mass in CMS.

A recent preliminary result [22] exploits a pure sample of t $\bar{t}$ events in the dilepton channel to measure the W-boson leptonic branching fractions. The $p_{\mathrm{T}}$ spectra of training leptons in ee, $\mathrm{e} \mu$ and $\mu \mu$ events are used to disentangle prompt $\mathrm{W} \rightarrow \mathrm{e} / \mu$ from $\mathrm{W} \rightarrow \tau \rightarrow \mathrm{e} / \mu$ decays. Despite being as precise as and consistent with, the CMS results do not confirm the slight departure $(2.5 \sigma)$ from lepton universality implied by LEP combined results.

The inclusive diboson production cross-sections ( $\mathrm{pp} \rightarrow \mathrm{WW}, \mathrm{WZ}$ and $\mathrm{ZZ}$ ) are now measured with 3-10\% precision at $\sqrt{s}=7,8$ and $13 \mathrm{TeV}$, and recently at $5.02 \mathrm{TeV}$ [23]. The excellent agreement with NNLO (QCD) and NLO (EWK) calculations puts stringent limits on anomalous triple gauge couplings. An abundant and pure sample of events with full Run-2 data is used to observe, for the first time, longitudinal polarisation of $\mathrm{W}$ bosons in $\mathrm{WZ}$ production [24]. There is conclusive signals of vector boson scattering (VBS) in all $\mathrm{VV}^{\prime} j j$ final states $\left(\mathrm{V}^{(\prime)}=\gamma, \mathrm{W}\right.$ or Z) [25-27]. Exploiting event kinematics to study the polarisation components in same-sign W pair VBS production, CMS reports a first hint (2.3 $\sigma$ obs., $3.1 \sigma$ exp.) of the scattering of at least one $\mathrm{W}_{\mathrm{L}}$ boson [25]. This is an important step towards probing unitarity restoration in longitudinal boson scattering at the electroweak symmetry breaking energy scale. 


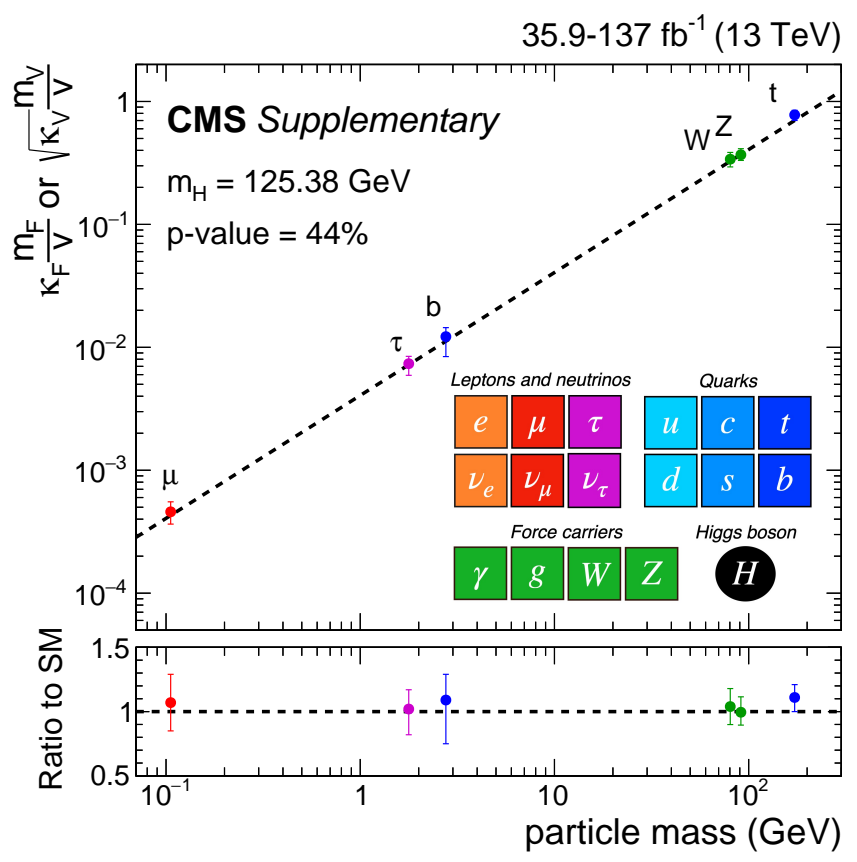

Figure 1: Reduced Higgs couplings as a function of particle mass. The points and error bars represent central values and $68 \% \mathrm{CL}$ intervals, respectively. The dashed line indicates the standard model prediction. The coupling with the muon, measured for the first time, is shown in red. The lower panel shows ratios of measured values to standard model predictions. Within the used $\kappa$-framework model, the data are consistent with the standard model predictions with a $p$-value of 44\%. From [28].

\subsection{Highlights on Higgs boson studies}

The Higgs-boson mass measurements in the $\mathrm{Z} \rightarrow 4 \ell$ [29] and $\mathrm{H} \rightarrow \gamma \gamma$ [30] decay modes with Run-2/2016 data are independently consistent with and more precise than the Run-1 combination. The overall combination, $m_{\mathrm{H}}=125.38 \pm 0.14 \mathrm{GeV}$, most precise to date, is used consistently in all CMS analyses. The $0.11 \%$ total uncertainty remains dominated by statistics.

The study of Higgs couplings intensifies, in the context of the Simplified Template Cross Section (STXS) framework, in particular in the $\gamma \gamma$ [31] and $\mathrm{ZZ}^{*}[32,33]$ decay modes. The kinematic complementarity of the two modes is exploited to study for the first time the CP structure of the $\mathrm{t} \overline{\mathrm{t}} \mathrm{H}$ coupling [34]. The recent evidence for the decay into muons [28] allows to establish for the first time meaningful $68 \%$ and $95 \%$ confidence intervals for a Higgs boson coupling to a second generation fermion (Fig. 1).

Impressive progress is made on the road to the observation of double-Higgs production. Limits at the $95 \% \mathrm{CL}$ are now at 7.7 obs. (5.2 exp.) times the standard model expectation in the $\mathrm{b} \bar{b} \gamma \gamma$

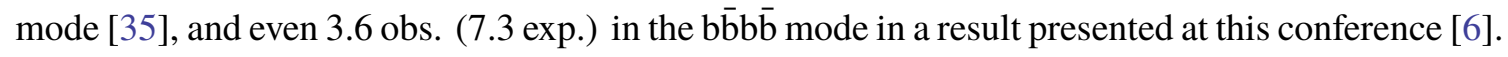

\subsection{Highlights on top-quark mass measurements}

The top-quark mass is a crucial fundamental parameter of the standard model. To complement the measurements in the main $t \bar{t}$ lepton+jets and dilepton channels, many alternative methods are developed, with different sources of systematic uncertainties. An example is the measurement of the 
top-quark mass in single-top events, which is quite precise in itself ( $0.77 \mathrm{GeV}$ in total uncertainty) and in addition allows separate measurements for the quark and antiquark [36]. Progress has been made also in extracting the top-quark pole mass from the comparison of inclusive and multidifferential $t \bar{t}$ cross section measurements with NLO predictions in the $\overline{\mathrm{MS}}$ scheme [37]. Using similar techniques, the measurement was performed in four bins of $m_{\bar{t} \mathrm{t}}$, establishing the running of the top-quark mass up to a scale of $1 \mathrm{TeV}$, in good agreement with RGE predictions [38].

\subsection{Highlights on heavy quarks studies in heavy ion collision}

The production of heavy quarks in lead-lead collisions is also studied. Following a previous observation in proton-lead collisions at $\sqrt{s_{\mathrm{NN}}}=8.16 \mathrm{TeV}$, CMS now reports evidence for tī production in lead-lead collisions at $\sqrt{s_{\mathrm{NN}}}=5.02 \mathrm{TeV}$ at $4.0 \sigma$ obs. (6.0 $\sigma$ exp.) [39]. Because they decay before QGP formation, top quarks are complementary to other probes and constitute a potential tool to study parton energy loss in the unconfined medium.

The $\mathrm{B}_{\mathrm{c}}$ production, observed with $\gg 5 \sigma$ in the $\mathrm{B}_{\mathrm{c}} \rightarrow \mathrm{J} / \psi(\rightarrow \mu \mu) \mu \nu$ decay mode as a bridge between charm and charmonium, provides a new tool to help disentangle enhancement and suppression mechanisms at play in the evolution of heavy quarks through the QGP [14]. The nuclear modification factor, measured in two $p_{\mathrm{T}}$ bins, already excludes some of the most extreme models of charm recombination.

\subsection{Highlights of beyond the standard model searches}

Searches for the production of new heavy particles promptly decaying to pairs of leptons [40] or vector bosons [41] with the full Run-2 data set, have been performed. In the latter case, one of the bosons is a $\mathrm{W}$ decaying leptonically, while the other one $(\mathrm{W}, \mathrm{Z}$ or $\mathrm{H})$ is boosted and detected as a merged jet with substructures. Many other searches are exploiting the novel boosted boson or top-quark detection techniques (for instance [17, 20, 42]). CMS has also developed a systematic search for deviations from the standard model predictions [41] in several hundred final states and kinematic distributions, in events containing at least one lepton.

CMS pursues a rich programme of search for super-symmetry, setting strong limits on many models. Most recent analyses are optimised to tackle difficult corners of the phase space (stop corridor, compressed scenarios), as for instance [43-45].

Since recently, lots of efforts go into searches for long-lived massive particles (LLPs) [19, 46]. One of these, presented at the conference, exploits special low threshold displaced dimuon triggers reconstructed at HLT level, called "scouting data", to set limits on scalar particles from B-meson decays or dark photons from Higgs-boson decays [18].

\section{Medium and long-term perspectives}

CMS is preparing for the Run-3 of the LHC, which will last three years (2022-2024), with, among other physics goals, improved precision on Higgs coupling measurements, observation of the $\mathrm{H} \rightarrow \mu^{+} \mu^{-}$and $\mathrm{H} \rightarrow \mathrm{Z} \gamma$ ultra-rare decays, and progress towards the observation of double-Higgs production. The trigger strategy has been refined in order to be sensitive to new exotic topologies, as displaced muons from LLP decays. The computing performances have been considerably 
improved, with, noticeably, the increased use of opportunistic high-performance computing and the deployment of GPUs to complement CPUs in the online compute farm.

For the longer term, CMS is actively working on upgrade projects for the high-luminosity phase- 2 of the LHC, known as HL-LHC. The experimental challenges of HL-LHC are formidable. The increase in instantaneous luminosity will result in up to five-fold pile-up and radiation levels. The phase-2 upgrades of the CMS detector are designed to mitigate this unprecedented experimental environment, with the goal of integrating 3 to $5,000 \mathrm{fb}^{-1}$ of high-quality $\sqrt{s}=14 \mathrm{TeV}$ pp collision data (2027-2038). The key elements of the CMS phase-2 upgrade programme are:

- a completely new central tracker, again all-silicon but with much higher granularity ( 2 billion pixels and micro-strips), a coverage extended to $\eta=4$ and level- 1 track-trigger capability;

- a new high-granularity calorimeter between 1.5 and 3 in pseudo-rapidity (HGCAL), which will sample EM-showers every radiation length (28 samples) with small silicon pixels, and hadron showers every 0.35 absorption length ( 24 samples) with a combination of silicon pixels and scintillating tiles, mapping the full development of all showers (a total of $6 \mathrm{M}$ channels) in 3 dimensions;

- precision timing on all particles, either neutral (ECAL and HGCAL) or charged (Mip Timing Detector, or MTD), providing reconstruction with a fourth dimension in order to mitigate the effects of pile-up;

- an improved trigger system, exploiting specialised processors (FPGAs at level-1, GPUs at HLT), with an output rate of $7.5 \mathrm{kHz}$ (compared to $1 \mathrm{kHz}$ in Run-2).

With this ambitious programme, described in detail elsewhere in these proceedings, CMS will not only be able to maintain the existing performance in the new, much harsher conditions at HL-LHC, but will even out-perform the current detector with new capabilities (for example, extended tracking acceptance or particle identification), which will significantly improve the physics reach of the experiment.

\section{Acknowledgments}

The author wishes to congratulate the organisers of LHCP 2021 for a particularly successful conference. He would like to thank all his CMS colleagues, as well as CERN and the accelerator department for the outstanding performance of the LHC. 


\section{References}

[1] CMS Collaboration, “ The CMS experiment at the CERN LHC”, JINST 3 (2008) S08004, doi : / $10.1088 / 1748-0221 / 3 / 08 /$ S08004.

[2] CMS Collaboration, "Precision luminosity measurement in proton-proton collisions at $\sqrt{s}=$ $13 \mathrm{TeV}$ in 2015 and 2016 at CMS", EPJC 81 (2021) 800, doi:10.1140/epjc/s10052-021-09538-2, arXiv:2104.01927.

[3] CMS Collaboration, "Luminosity measurement in proton-proton collisions at $5.02 \mathrm{TeV}$ in 2017 at CMS”, CMS-PAS-LUM-19-001 (2021). https://cdsweb.cern.ch/record/2765655.

[4] CMS Collaboration, "Measurement of multi-differential cross sections for the production of a $\mathrm{Z}$ boson in association with jets in proton-proton collisions at $\sqrt{s}=13 \mathrm{TeV}$ ", CMS-PAS-SMP-19-009 (2021). https: //cdsweb. cern. ch/record/2767703.

[5] CMS Collaboration, "Measurement of mass dependence of the transverse momentum of Drell Yan lepton pairs in proton-proton collisions at $\sqrt{s}=13 \mathrm{TeV}$ ", CMS-PAS-SMP-20-003 (2021). https://cdsweb. cern.ch/record/2764470.

[6] CMS Collaboration, "Search for Higgs boson pair production in the four b quark final state", CMS-PAS-HIG-20-005 (2021). https: //cdsweb . cern. ch/record/2771912.

[7] CMS Collaboration, "Measurement of the inclusive and differential Higgs boson production cross sections in the decay mode to a pair of $\tau$ leptons in pp collisions at $\sqrt{s}=13 \mathrm{TeV}$ ", arXiv:2107 . 11486. Submitted to PRL.

[8] CMS Collaboration, "Measurement of the shape of the $b$ quark fragmentation function using charmed mesons produced inside b jets from tt̄ pair decays", CMS-PAS-TOP-18-012 (2021). https://cdsweb.cern.ch/record/2771694.

[9] CMS Collaboration, "Search for charged lepton flavor violation in top quark production and decay in proton-proton collisions at $\sqrt{s}=13 \mathrm{TeV}$ ", CMS-PAS-TOP-19-006 (2021). https://cdsweb. cern. ch/record/2771682.

[10] CMS Collaboration, "Search for flavor-changing neutral current interactions of the top quark and the Higgs boson in the diphoton decay channel in proton-proton collisions at $\sqrt{s}=13$ TeV", CMS-PAS-TOP-20-007 (2021). https://cdsweb. cern.ch/record/2771683.

[11] CMS Collaboration, "Inclusive and differential cross section measurements of single top quark production in association with a $\mathrm{Z}$ boson in proton-proton collisions at $\sqrt{s}=13 \mathrm{TeV}$ ", CMS-PAS-TOP-20-010 (2021). https: //cdsweb . cern. ch/record/2771809.

[12] CMS Collaboration, "Probing effective field theory operators in the associated production of top quarks with a Z boson in multilepton final states at $\sqrt{s}=13 \mathrm{TeV}$ ", arXiv:2107.13896. Submitted to JHEP. 
[13] CMS Collaboration, "Strange particle collectivity in $\mathrm{pPb}$ and $\mathrm{PbPb}$ ", CMS-PAS-HIN-19-004 (2021). https: //cdsweb. cern. ch/record/2767034.

[14] CMS Collaboration, "Observation of the $\mathrm{B}_{\mathrm{c}}^{+}$meson in $\mathrm{PbPb}$ and pp collisions at $\sqrt{s_{\mathrm{NN}}}=5.02$ TeV”, CMS-PAS-HIN-20-004 (2021). https://cdsweb.cern.ch/record/2767048.

[15] CMS Collaboration, "Search for Higgs boson decays into long-lived particles in associated Z boson production”, CMS-PAS-EXO-20-003 (2021).

https://cdsweb. cern.ch/record/2767507.

[16] CMS Collaboration, "Search for new particles in events with energetic jets and large missing transverse momentum in proton-proton collisions at $\sqrt{s}=13 \mathrm{TeV}$ ", arXiv:2107.13021. Submitted to JHEP.

[17] CMS Collaboration, "Search for high mass trijet resonances using final states with boosted dijet resonances in proton-proton collisions at $\sqrt{s}=13 \mathrm{TeV}$ ", CMS-PAS-EXO-20-007 (2021). https://cdsweb. cern. ch/record/2771810.

[18] CMS Collaboration, "Search for long-lived particles decaying into two muons in proton-proton collisions at $\sqrt{s}=13 \mathrm{TeV}$ using data collected with high rate triggers", CMS-PAS-EXO-20-014 (2021). https: //cdsweb . cern. ch/record/2767659.

[19] CMS Collaboration, "Search for long-lived particles decaying in the CMS endcap muon detectors in proton-proton collisions at $\sqrt{s}=13 \mathrm{TeV}$ ", arXiv:2107.04838. Submitted to PRL.

[20] CMS Collaboration, "Search for resonances decaying to triple W-boson final states in proton-proton collisions at $\sqrt{s}=13 \mathrm{TeV}$ ", CMS-PAS-B2G-20-001 (2021). https://cdsweb.cern.ch/record/2759857.

[21] CMS Collaboration, "Measurements of the $\mathrm{W}$ boson rapidity, helicity, double-differential cross sections, and charge asymmetry in pp collisions at 13 TeV", PRD 102 (2020) 092012, doi:10.1103/PhysRevD.102.092012, arXiv:2008.04174.

[22] CMS Collaboration, "A precision measurement of the $\mathrm{W}$ boson decay branching fractions in pp collisions at $\sqrt{s}=13 \mathrm{TeV}$ ", CMS-PAS-SMP-18-011 (2021). https://cdsweb. cern.ch/record/2758905.

[23] CMS Collaboration, "Measurements of the electroweak diboson production cross sections in proton-proton collisions at $\sqrt{s}=5.02 \mathrm{TeV}$ using leptonic decays", arXiv : 2107.01137. Accepted by PRL.

[24] CMS Collaboration, "Measurement of the pp $\rightarrow$ WZ inclusive and differential cross sections, polarization angles and search for anomalous gauge couplings at $\sqrt{s}=13 \mathrm{TeV}$ ", CMS-PAS-SMP-20-014 (2021). https : //cdsweb . cern. ch/record/2758362.

[25] CMS Collaboration, "Measurements of production cross sections of polarized same-sign W boson pairs in association with two jets in proton-proton collisions at $\sqrt{s}=13 \mathrm{TeV}$ ", PLB 812 (2020) 136018, doi: $10.1016 / \mathrm{j}$. physletb. 2020 . 136018, arXiv: 2009.09429. 
[26] CMS Collaboration, "Observation of electroweak production of $\mathrm{W} \gamma$ with two jets in proton-proton collisions at $\sqrt{s}=13 \mathrm{TeV}$ ", PLB 811 (2020) 135988, doi:10.1016/j.physletb.2020.135988, arXiv:2008.10521.

[27] CMS Collaboration, "Evidence for electroweak production of four charged leptons and two jets in proton-proton collisions at $\sqrt{s}=13 \mathrm{TeV}$ ", PLB 812 (2020) 135992, doi:10.1016/j.physletb.2020.135992, arXiv:2008.07013.

[28] CMS Collaboration, "Evidence for Higgs boson decay to a pair of muons", JHEP 01 (2021) 148, doi : 10.1007/JHEP01(2021) 148, arXiv: 2009.04363.

[29] CMS Collaboration, "Measurements of properties of the Higgs boson decaying into the four-lepton final state in pp collisions at $\sqrt{s}=13 \mathrm{TeV}$ ", JHEP 11 (2017) 047, doi : 10 . 1007/JHEP11(2017)047, arXiv: 1706.09936.

[30] CMS Collaboration, "A measurement of the Higgs boson mass in the diphoton decay channel”, PLB 805 (2020) 135425, doi:10.1016/j . physletb.2020.135425, arXiv:2002.06398.

[31] CMS Collaboration, "Measurements of Higgs boson production cross sections and couplings in the diphoton decay channel at $\sqrt{s}=13 \mathrm{TeV}$ ", JHEP 07 (2021) 027, doi : 10.1007/JHEP07 (2021)027, arXiv: 2103.06956.

[32] CMS Collaboration, "Measurements of production cross sections of the Higgs boson in the four-lepton final state in proton-proton collisions at $\sqrt{s}=13 \mathrm{TeV}$ ", EPJC 81 (2021) 488, doi:10.1140/epjc/s10052-021-09200-x, arXiv:2103.04956.

[33] CMS Collaboration, "Measurement of the Higgs boson production rate in association with top quarks in final states with electrons, muons, and hadronically decaying tau leptons at $\sqrt{s}=13 \mathrm{TeV} "$, EPJC 81 (2021) 378, doi : 10.1140/epjc/s10052-021-09014-x, arXiv:2011.03652.

[34] CMS Collaboration, "Constraints on anomalous Higgs boson couplings to vector bosons and fermions in its production and decay using the four-lepton final state", PRD 104 (2021) 052004, doi: 10.1103/PhysRevD. 104.052004, arXiv:2104.12152.

[35] CMS Collaboration, "Search for nonresonant Higgs boson pair production in final states with two bottom quarks and two photons in proton-proton collisions at $\sqrt{s}=13 \mathrm{TeV}$ ", JHEP 03 (2021) 257, doi : JHEP03(2021)257, arXiv:2011.12373.

[36] CMS Collaboration, "Measurement of the top quark mass using events with a single reconstructed top quark in pp collisions at $\sqrt{s}=13 \mathrm{TeV}$ ", arXiv: 2108.10407. Submitted to JHEP.

[37] CMS Collaboration, "Measurement of ț̄ normalised multi-differential cross sections in pp collisions at $\sqrt{s}=13 \mathrm{TeV}$, and simultaneous determination of the strong coupling strength, top quark pole mass, and parton distribution functions", EPJC 80 (2020) 658, doi:10.1140/epjc/s10052-020-7917-7, arXiv: 1904.05237. 
[38] CMS Collaboration, "Running of the top quark mass from proton-proton collisions at $\sqrt{s}=$ 13 TeV”, PLB 803 (2020) 135263, doi:10.1016/j .physletb.2020.135263, arXiv: 1909.09193.

[39] CMS Collaboration, "Evidence for top quark production in nucleus-nucleus collisions", PRL 125 (2020) 222001, doi : 10.1103/PhysRevLett.125.222001, arXiv:2006.11110.

[40] CMS Collaboration, "Search for resonant and nonresonant new phenomena in high-mass dilepton final states at $\sqrt{s}=13 \mathrm{TeV}$ ", JHEP 07 (2021) 208, doi : 10.1007/JHEP07 (2021)208, arXiv:2103.02708.

[41] CMS Collaboration, "MUSiC: a model unspecific search for new physics in proton-proton collisions at $\sqrt{s}=13 \mathrm{TeV}$ ", EPJC 81 (2021) 629, doi : 10.1140/epjc/s10052-021-09236-z, arXiv:2010.02984.

[42] CMS Collaboration, "Search for heavy resonances decaying to WW, WZ, or WH boson pairs in the lepton plus merged jet final state in proton-proton collisions at $\sqrt{s}=13 \mathrm{TeV}$ ", arXiv:2109.06055. Submitted to PRD.

[43] CMS Collaboration, "Combined searches for the production of supersymmetric top quark partners in proton-proton collisions at $\sqrt{s}=13 \mathrm{TeV}$ ", arXiv:2107.10892. Submitted to EPJC.

[44] CMS Collaboration, "Search for physics beyond the standard model in final states with two or three soft leptons and missing transverse momentum in proton-proton collisions at 13 TeV", CMS-PAS-SUS-18-004 (2021). https: //cdsweb. cern.ch/record/2758359.

[45] CMS Collaboration, "Search for disappearing tracks in proton-proton collisions at $\sqrt{s}=13$ TeV”, PLB 806 (2020) 135502, doi:10.1016/j . physletb.2020.135502, arXiv:2004.05153.

[46] CMS Collaboration, "Search for long-lived particles decaying to jets with displaced vertices in proton-proton collisions at $\sqrt{s}=13 \mathrm{TeV}$ ", arXiv : 2104.13474. Accepted by PRD. 